\title{
Infantile hydrocephalus epidemiology: an indicator of enhanced survival
}

Department of Paediatrics II University of Gothenburg, Sweden G Hagberg B Hagberg

Section of Neuropaediatrics, Department of Paediatrics, Huddinge Hospital S-141 86, Huddinge, Sweden E Fernell

Correspondence to: Dr Fernell.

Accepted 6 September 1993

\author{
E Fernell, G Hagberg, B Hagberg
}

\begin{abstract}
The epidemiology of infantile hydrocephalus in the birth years 1973-90 was investigated in west Sweden. The study revealed a significant increase in prevalence from 1973-8 and 1979-82 considered due to the enhanced survival of very and extremely preterm infants. The increase did not continue from 1983-90. This could indicate an improved outcome in preterm survivors as the neonatal survival rate continued to increase. The striking predominance of a perinatal/neonatal aetiology in very preterm hydrocephalic infants could be confirmed: $89 \%$ born from 1983-90 had suffered a confirmed postpartum intraventricular haemorrhage. In infants born at term, prenatal origins, mainly maldevelopments, dominated. The outcome in very preterm surviving infants with infantile hydrocephalus was poor: $73 \%$ had cerebral palsy, $52 \%$ epilepsy, $22 \%$ severe visual disability, and $55 \%$ were mentally retarded. Despite the increased survival resulting in a majority of healthy infants, there is an accumulating cohort of hydrocephalic children.
\end{abstract}

(Arch Dis Child 1994; 70: F123-F128)

Systematic epidemiological and clinical follow up studies of children with prenatally and perinatally/neonatally acquired brain impairments are important as feedback to neonatologists. This applies especially to very preterm infants, a group in which the increase in survival has been striking during the last few decades and the risk of perinatal vascular insult is marked. In a previous series of studies, we have suggested the epidemiology of cerebral palsy as an instrument for such feedback. ${ }^{1}$ However, cerebral palsy has some obvious disadvantages. Firstly, there is disagreement about the timing of brain damaging events resulting in cerebral palsy in the very preterm infant and, secondly, a time period of at least four years is needed before a definite clinical cerebral palsy diagnosis can be settled in all cases. In view of both these aspects, infantile hydrocephalus might be a better alternative for rapid feedback to neonatalogists. In around $90 \%$ of very preterm infants, infantile hydrocephalus is undisputedly acquired perinatally/neonatally and can be diagnosed already during the first weeks of life, virtually always before 6 months of age. ${ }^{2}$ One disadvantage is that infantile hydrocephalus is a rare condition, present in only some $2 \%$ of surviving very preterm infants, thereby making large populations necessary for any conclusions to be drawn.

This presentation is the third in an ongoing infantile hydrocephalus project in west Sweden, previously comprising birth years $1967-86,{ }^{2} 3$ and now extended to those born in 1987-90. The main aims were (1) to follow, on a population basis, the trends in prevalence, the pattern of aetiologies, and the long term outcome from 1973 to 1990 , a period of time in which neonatal intensive care had been introduced and gestational age specific vital statistics were available, and (2) to try to provide informative points for feedback to neonatology.

\section{Definitions}

Infantile hydrocephalus was considered in cases with the following three main criteria. ${ }^{1}$ Indications of increased intraventricular pressure in terms of (a) insertion of an intraventricular shunt before the age of 1 year; (b) head circumference at birth of $>2$ SD above the mean both for gestational age and for birth length ${ }^{3}$; or (c) increment in head circumference of $>2$ SD for the age during the first year of life, concurrent with a head circumference of $>2$ SD above the mean for actual height. $^{3}$ (2) Indication of increased intraventricular cerebrospinal fluid volume in terms of ventricular dilatation amounting to a Sjögren's echo index $x^{4}$ of $\geqslant 0.39$ or a comparable value based upon ultrasonic or computerised examinations. (3) An infantile hydrocephalus duration of at least three months in the first year of life, in conservatively treated cases. All children with infantile hydrocephalus due to neural tube defects or intracranial malignant tumours were excluded.

Very preterm referred to infants born before gestational age of 32 completed weeks and moderately preterm to those born between the 32nd and 36th completed weeks of gestation. Prenatal referred to the period before the onset of labour resulting in delivery, perinatal from the onset of labour resulting in delivery to the seventh day of life. Neonatal referred to the first 28 days of life and postnatal to the period from the 29th day of life to the age of 1 year. A prenatal aetiology was considered in cases of genetically determined infantile hydrocephalus, cytogenetic abnormality, specific central nervous system maldevelopment, confirmed intrauterine infection, and in other cases in which infantile hydrocephalus was present already at birth. Perinatalneonatal and postnatal aetiologies were considered in cases of confirmed intraventricular haemorrhage (IVH) 
and confirmed central nervous system (CNS) infection in the perinatal/neonatal and postnatal periods respectively. Because of the well documented high association of perinatal/ neonatal IVH and CNS infection to the development of infantile hydrocephalus, a perinatal/neonatal aetiology was given precedence in case of overlapping between prenatal and perinatal/neonatal origin. Mental retardation was defined in school age children as an IQ of $\leqslant 70$ and as marked developmental delay in younger ages, cerebral palsy was defined as 'a disorder of movement and posture due to a defect or lesion of the immature brain', 5 and epilepsy was considered to be present whenever there was an undisputed diagnosis of epilepsy in the medical records.

\section{Subjects and methods}

The study area was the western Swedish health care region as defined previously ${ }^{3}$ with a total population of 1.9 million, $23 \%$ of the total population of Sweden. The study period comprised birth years 1973-90 with 420856 live births.

The identification of cases of infantile hydrocephalus was based upon the assumption that all the children with this condition would have been referred to a paediatric clinic and registered with a diagnosis of hydrocephalus according to the international classification system (ICD 1968, 1987). Diagnostic registers at all the departments of paediatrics in the investigated region and at the single regional neurosurgical unit at the University of Gothenburg were therefore scrutinised in terms of the diagnosis of infantile hydrocephalus. All children fulfilling the defined criteria for infantile hydrocephalus and living in the region at birth were included in the series. Information relating to gestational age, birth weight, aetiology of infantile hydrocephalus, and any additional major impairments was obtained from obstetric, paediatric, and neurosurgical records. The mean age of children at evaluation was 5.5 years (range 3-12).

Information on vital statistics was provided by the Medical Birth Registration, Statistics of the National Board of Health and Welfare, Sweden. The neonatal mortality in the study population decreased successively during the periods investigated as shown in fig 1 ; in the very preterm group from 343 per 1000 in

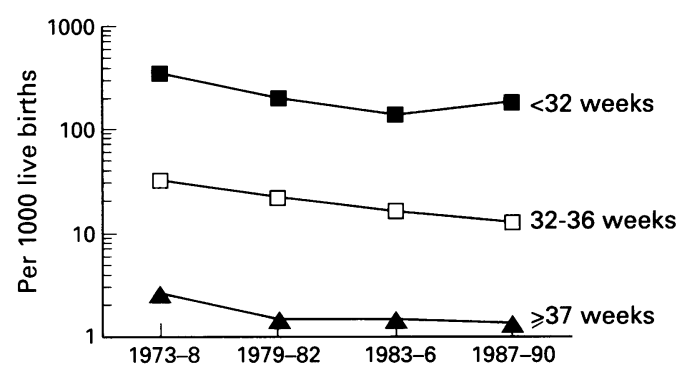

Figure 1 Gestational age specific neonatal mortality in west Sweden from 1973-1990.
$1973-8$ to 184 per 1000 in $1987-90$, that is by $46 \%$. For statistical calculations the $\chi^{2}$ test for contingency tables and trends in proportions was used. ${ }^{6}$

\section{Results}

This population based study comprised 251 liveborn infants born in 1973-90 and fulfilling our criteria for infantile hydrocephalus. Of these, 57 were born very preterm $(23 \%), 46$ moderately preterm (18\%), and 148 were born at term $(59 \%)$. The median gestational age in the very preterm group was 30 weeks in the period 1973-8, 28 in 1979-82, 28 in 1983-6, and 29 in $1987-90$. Of the 251 patients, 211 $(84 \%)$ had required an intraventricular shunt, $19(8 \%)$ had died without a shunt insertion, and $21(8 \%)$ were conservatively treated and considered to have arrested infantile hydrocephalus.

\section{LIVE BIRTHS}

Prevalence

The mean crude prevalence of infantile hydrocephalus was 0.60 per 1000 live births; the mean gestational age specific prevalence was $17 \cdot 36$ per 1000 very preterm infants, 2.24 per 1000 moderately preterm, and 0.37 per 1000 infants born at term. The gestational age
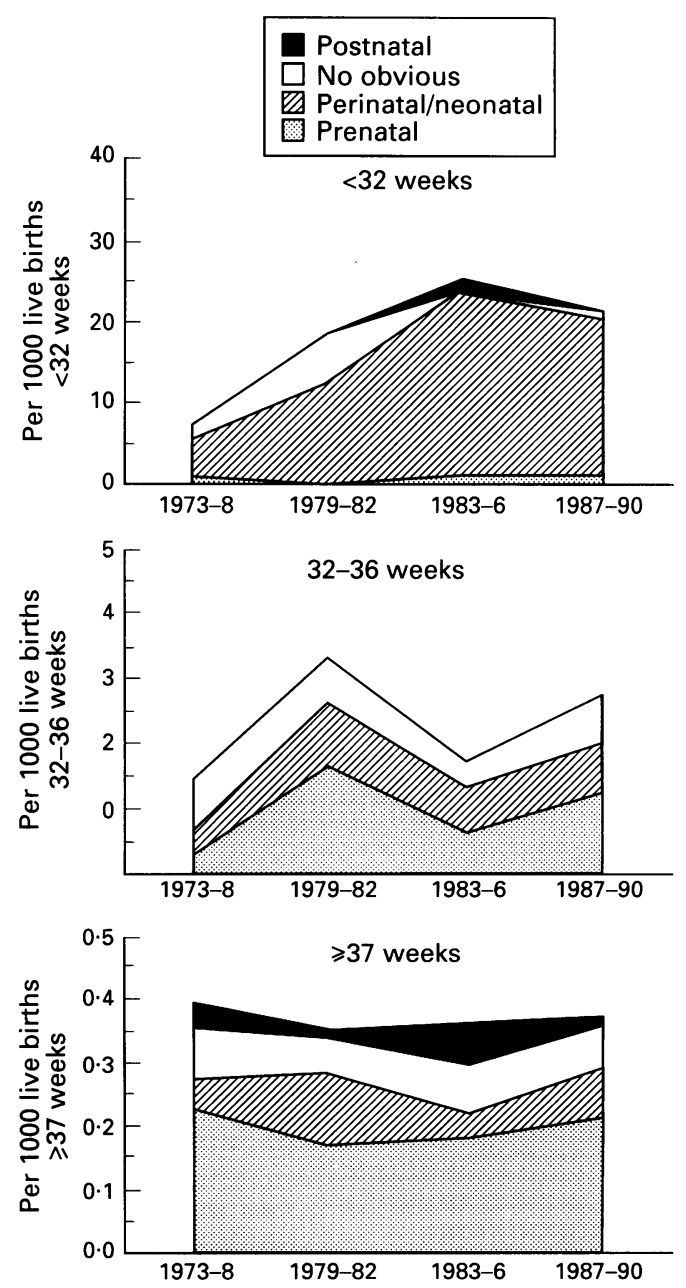

Figure 2 Gestational age specific live birth prevalence of infantile hydrocephalus in west Sweden from 1973-1990 according to aetiology. 
Table 1 The maldevelopments behind prenatal infantile hydrocephalus in the present series by gestational age

\begin{tabular}{llll}
\hline & \multicolumn{3}{c}{ Gestational age (weeks) } \\
\cline { 2 - 4 } & $\geqslant 37$ & $32-36$ & $<32$ \\
\hline Inherited & 2 & 0 & 0 \\
$\quad$ X linked aqueductstenosis & 3 & 0 & 0 \\
$\quad$ Other & 1 & $1 \ddagger$ & 1 \\
Chromosomal major aberration & & & \\
Prenatal syndrome & 6 & 1 & $1 \ddagger$ \\
$\quad$ Defined syndrome & 2 & 2 & 0 \\
$\quad$ Dandy-Walker and allied & $19(3 \ddagger)$ & $8(5 \ddagger)$ & $1 \ddagger$ \\
Multiple malformations & 2 & 4 & 0 \\
Hydranencephaly & 4 & 1 & 0 \\
Prenatal isolated cystic & 3 & 0 & 0 \\
$\quad$ Arachnoidal & 4 & 1 & 0 \\
Porencephalic & 20 & 1 & 0 \\
Other & 66 & 19 & 3 \\
Simple infantile hydrocephalus & & & \\
Total & &
\end{tabular}

*Trisomy $9(n=1)$, trisomy $18(n=1)$, translocation $7: 18$ $(\mathrm{n}=1)$.

†Carpenter's $(n=1)$, Crouzon's ( $n=4)$, Noonan's $(n=1)$, Warburg's $(n=1)$, fetal alcohol syndrome $(n=1)$.

tNeonatal deaths.

specific prevalence of infantile hydrocephalus per live births according to birth year period and aetiology is shown in fig 2 . In very preterm infants, the prevalence of infantile hydrocephalus increased significantly during the periods $1973-8$ to $1983-6$, from 6.99 to $25 \cdot 37$ per $1000(\mathrm{p}<0.01)$, and then decreased slightly 21.47 per 1000 . The increase was entirely due to a higher prevalence of very preterm infants with a perinatal/neonatal aetiology of infantile hydrocephalus. A prenatal and postnatal aetiology was seen only in exceptional very preterm cases (three cases and one case respectively). The group with no obvious origin of infantile hydrocephalus decreased over time and occurred in only one very preterm infant during the two periods investigated most recently, 1983-90. In the moderately preterm and full term groups, the live birth prevalence did not reveal any significant change in temporal trends, either in overall terms or within the different aetiological groups.

\section{Neonatal deaths}

A total of 12 infants with infantile hydrocephalus $(5 \%)$ died in the neonatal period, 2/57 very preterm, $6 / 46$ moderately preterm,

Table 2 Distribution of aetiology in 239 neonatally surviving infants with infantile hydrocephalus according to birth year period and gestational age; data are number (\%)

\begin{tabular}{lllllr}
\hline & Prenatal & $\begin{array}{l}\text { Perinatal } \\
\text { neonatal }\end{array}$ & Postnatal & No obvious & Total \\
\hline$<32$ weeks & & & & & \\
$1973-8$ & 0 & $4(67)$ & 0 & $2(33)$ & $6(100)$ \\
$1979-82$ & 0 & $8(67)$ & 0 & $4(33)$ & $12(100)$ \\
$1983-6$ & $1(5)$ & $17(90)$ & $1(5)$ & 0 & $19(100)$ \\
$1987-90$ & 0 & $17(94)$ & 0 & $1(6)$ & $18(100)$ \\
Subtotal & $1(2)$ & $46(84)$ & $1(2)$ & $7(13)$ & $55(100)$ \\
$32-36$ weeks & & & & & \\
$1973-8$ & 0 & $2(29)$ & 0 & $5(71)$ & $7(100)$ \\
$1979-82$ & $6(46)$ & $4(31)$ & 0 & $3(23)$ & $13(100)$ \\
$1983-6$ & $2(29)$ & $3(43)$ & 0 & $2(29)$ & $7(100)$ \\
$1987-90$ & $5(38)$ & $4(31)$ & 0 & $13(100)$ \\
Subtotal & $13(33)$ & $13(33)$ & 0 & $14(35)$ & $40(100)$ \\
$\geqslant 37$ weeks & & & & & \\
$1973-8$ & $26(51)$ & $5(10)$ & $4(8)$ & $16(31)$ & $51(100)$ \\
$1979-82$ & $10(34)$ & $4(14)$ & $1(3)$ & $14(48)$ & $29(100)$ \\
$1983-6$ & $13(46)$ & $3(11)$ & $5(18)$ & $7(25)$ & $28(100)$ \\
$1987-90$ & $20(56)$ & $8(22)$ & $1(3)$ & $7(19)$ & $36(100)$ \\
Subtotal & $69(48)$ & $20(14)$ & $11(8)$ & $44(31)$ & $144(100)$ \\
Total & $83(35)$ & $79(33)$ & $12(5)$ & $65(27)$ & $239(100)$ \\
\hline
\end{tabular}

and $4 / 148$ full term. Five of these deaths occurred in the earlier half of the period investigated, 1973-82, and seven in the latter half, 1983-90. All 12 neonatally dead infants had a prenatal aetiology of infantile hydrocephalus, with severe multiple malformations in 11 (table 1) and intrauterine toxoplasmosis in one.

\section{NEONATAL SURVIVORS}

\section{Prevalence}

The crude prevalence of infantile hydrocephalus was 0.57 per 1000 neonatal survivors; the gestational age specific prevalence was 21.65 per 1000 very preterm infants, 1.99 per 1000 moderately preterm, and 0.36 per 1000 infants born at term. The prevalence per 1000 neonatally surviving very preterm infants increased significantly during the periods 1973-8 to $1983-6$, from $9 \cdot 12$ to 29.64 per $1000(\mathrm{p}<0.01)$ and then decreased slightly to 24.93 per 1000 . The prevalence per 1000 neonatal survivors in the moderately preterm and full term groups did not reveal any significant change in temporal trends.

\section{Aetiology}

The distribution of aetiology in neonatally surviving children with infantile hydrocephalus is shown in table 2 . Overlap between aetiological grouping was only present in one case, a boy (gestational age 32 weeks) with an IVH grade IV and $60 \%$ mosaic trisomy 8 . He was classified as having a perinatal/neonatal origin of infantile hydrocephalus.

In the very preterm group, a perinatal/ neonatal aetiology of infantile hydrocephalus dominated and was present in 46 of 55 cases $(84 \%)$. Of these 46,45 had had an IVH and one listeria meningitis. In the last two periods, 1983-90, the origin of infantile hydrocephalus could be traced for 36 of 37 very preterm infants. Of these $36,34(94 \%)$ had a perinatal/neonatal, one a prenatal (chromosome translocation 7:18), and one a postnatal aetiology (Escherichia coli meningitis).

In the moderately preterm group, there was an equal distribution of aetiology, 33\% prenatal, $33 \%$ perinatal/neonatal, and $35 \%$ with no obvious origin of infantile hydrocephalus. Thirteen infants had maldevelopments of different types (table 1). Of the 13 infants with a perinatal/neonatal aetiology, 12 had had an IVH and one $\beta$ streptococci meningitis in the perinatal/neonatal period. The proportion of infants with no obvious origin of infantile hydrocephalus was highest in the earliest period 1973-8.

In the term group, a prenatal aetiology of infantile hydrocephalus dominated, comprising $48 \%$. A perinatal/neonatal aetiology was found in $14 \%$, a postnatal aetiology in $8 \%$, and no obvious origin of infantile hydrocephalus in $31 \%$. Of the 69 infants with a prenatal aetiology, 63 had maldevelopments of different types (table 1), and six were secondary to congenital infections (five toxoplasmosis and one parotitis). Of the 20 infants with a 
Table 3 Outcome in 239 neonatally surviving children with infantile hydrocephalus according to birth year period

\begin{tabular}{llllc}
\hline & \multicolumn{3}{l}{ Birth year period } & \\
\cline { 2 - 5 } & $\begin{array}{l}1973-8 \\
(n=64)\end{array}$ & $\begin{array}{l}1979-82 \\
(n=54)\end{array}$ & $\begin{array}{l}1983-6 \\
(n=54)\end{array}$ & $\begin{array}{l}1987-90 \\
(n=67)\end{array}$ \\
\hline Dead <2 years of age & 8 & 6 & 3 & 1 \\
Surviving ₹2 years of age & 56 & 48 & 51 & 66 \\
$\quad$ Without additional impairments & 31 & 22 & 20 & 27 \\
With additional impairments & 25 & 26 & 31 & 39 \\
\hline
\end{tabular}

perinatal/neonatal aetiology, 15 had had an IVH and five meningitis (one $E$ coli, two listeria, one klebsiella, and one virus of unknown type) in the perinatal/neonatal period.

\section{OUTCOME}

The outcome at 2 years of age according to birth year period is shown in table 3. Eighteen of the 239 neonatally surviving children had died before reaching 2 years of age $(8 \%)$. The proportion of deaths decreased significantly during the investigated period, from 8/64 $(13 \%)$ in $1973-8$ to $1 / 67(1 \%)$ in $1987-90$, $\mathrm{p}<0.01$. Of the 18 deaths, 14 had infantile hydrocephalus of prenatal origin, five born moderately preterm and nine at term. The remaining four had perinatal/neonatally acquired infantile hydrocephalus, all four born very preterm. The proportion of children with infantile hydrocephalus surviving to 2 years of age with major additional neuroimpairments increased significantly from $25 / 64(39 \%)$ in $1973-8$ to $39 / 67$ (58\%) in 1987-90, $\mathrm{p}<0.05$. The very preterm infantile hydrocephalus group comprised significantly more children with additional major neuroimpairments or postnatal deaths than the term group, 44/55 $(80 \%) v 69 / 144(48 \%), \mathrm{p}<0.001$ (table 4). This pattern was consistent through the birth year periods.

A prenatal or perinatal/neonatal aetiology of infantile hydrocephalus was associated with a poorer outcome than a postnatal cause or no obvious origin of infantile hydrocephalus. In the two most recently investigated periods 1983-90, when an obvious aetiology was present in $83 \%$ of cases, $68 \%(28 / 41)$ of the prenatal group were multi-impaired or had died before 2 years of age. The corresponding proportion in the perinatal/neonatal group was $71 \%(37 / 52)$, in the postnatal group $(43 \%$ $(3 / 7)$, and in the group with no obvious origin

Table 4 Outcome in 239 neonatally surviving children with infantile hydrocephalus according to gestational age; data are number (\%)

\begin{tabular}{llccc}
\hline & \multicolumn{4}{l}{ Gestational age (weeks) } \\
\cline { 2 - 5 } & $\begin{array}{l}<32 \\
(n=55)\end{array}$ & $\begin{array}{l}32-36 \\
(n=40)\end{array}$ & $\begin{array}{c}\geqslant 37 \\
(n=144)\end{array}$ & $\begin{array}{l}\text { Total } \\
(n=239)\end{array}$ \\
\hline Dead <2 years of age & 4 & 5 & 9 & 18 \\
Surviving $\geqslant 2$ years of age & $51(100)$ & $35(100)$ & $135(100)$ & $221(100)$ \\
$\quad$ Without additional impairments & $11(22)$ & $14(40)$ & $75(56)$ & $100(45)$ \\
$\quad$ With additional impairments & $40(78)$ & $21(60)$ & $60(44)$ & $121(55)$ \\
Cerebral palsy & $37(73)$ & $12(34)$ & $28(21)$ & $77(35)$ \\
Mental retardation & $28(55)$ & $16(46)$ & $36(27)$ & $80(36)$ \\
Epilepsy & $25(49)$ & $8(23)$ & $35(26)$ & $68(31)$ \\
Blind/near blind & $11(22)$ & $3(9)$ & $11(8)$ & $25(11)$ \\
Mean number of impairments in & & & & \\
$\quad$ survivors & 2.0 & $1 \cdot 1$ & 0.8 & $1 \cdot 1$ \\
\hline
\end{tabular}

of infantile hydrocephalus $29 \%(6 / 21)$. The poorest outcome was found in the very preterm group with a perinatal/neonatal aetiology in which $82 \%$ of cases $(28 / 34)$ were multiimpaired or had died before 2 years of age. The most common additional impairment in this group was cerebral palsy, present in 25 of 33 $(76 \%)$ surviving to years of age.

In overall terms, $35 \%$ of children with infantile hydrocephalus surviving to 2 years of age had cerebral palsy, $36 \%$ mental retardation, $31 \%$ epilepsy, and $11 \%$ severe visual impairment (table 4). Major additional neuroimpairments, which were severely disabling, were especially frequent in the very preterm group: $73 \%$ had cerebral palsy, $55 \%$ were mentally retarded, $49 \%$ had or had had epilepsy, and $22 \%$ were blind or near blind. The mean number of additional neuroimpairments in children surviving to 2 years of age was 1.1 in overall terms, 2.0 in the very preterm group, 1.1 in the moderately preterm, and 0.8 in the term group with no difference over time.

\section{Discussion}

The continuous improvement in perinatal care has resulted in an enhanced general survival rate, especially among the very preterm infants. There is no question that large cohorts of newborns, who would previously have died, are now saved for a healthy life. ${ }^{1}$ At the same time, however, suspicion has been raised that new care routines have kept alive infants who were severely brain damaged either prenatally or around birth, ${ }^{7}$ or may have had harmful effects which were not immediately apparent. So, for obstetricians and neonatologists, neuropaediatric long term follow up studies elucidating aetiological factors constitute an essential part of a neonatal intensive care service. In this respect our experience ${ }^{2} 8$ indicates that infantile hydrocephalus is one appropriate disorder to study as the vast majority of cases can be accurately classified in terms of aetiology. Increasing access to the rapidly developing neuroimaging techniques has given and will continue to provide greater potential for revealing the timing of brain damaging events, ${ }^{9}$ which in turn provides clues to the underlying mechanisms. Consequently, in the latter part of this study, comprising birth years 1983-90, an obvious origin of infantile hydrocephalus was settled in a total of $83 \%$ of cases; in the case of the very preterm group in all but one of 37 children.

The origins of infantile hydrocephalus are known to differ strikingly between children born very preterm and those born at term. ${ }^{2} 1011$ Every stage of brain maturation, that is ventral induction, neuronal proliferation and neuronal migration, can be associated with or parallel to infantile hydrocephalus. In addition, children born very preterm have a high frequency of intracranial compromise leading to IVH with the inherent risk of posthaemorrhagic infantile hydrocephalus. From about 24 to 32 weeks of fetal life there is a rich and fragile capillary bed in the subependymal terminal layer. These 
capillaries are highly susceptible to rupture. In the $1970 \mathrm{~s}$, the incidence of IVH in very preterm infants was reported to be as high as $40 \%-90 \% .{ }^{12}{ }^{13}$ During the 1980 s a decrease in the incidence was reported. ${ }^{14}$ In the period $1983-90,92 \%$ of our very preterm infants with infantile hydrocephalus had a perinatal/neonatal aetiology, all but one IVH. Infantile hydrocephalus in very preterm infants was therefore considered suitable as a group for analysis in order to elucidate the advances and risks of neonatal intensive care. In contrast, infantile hydrocephalus in more than half of the corresponding term group was prenatally derived and only $17 \%$ perinatally/neonatally acquired.

There are essentially two areas in which follow up studies can serve as feedback to neonatologists, trends in prevalence rates and the short and long term outcome. The group of very preterm infants with a perinatal/neonatal aetiology was entirely responsible for the demonstrated increase in infantile hydrocephalus prevalence. A more detailed analysis revealed that most of the increase occurred between the first two periods, 1973-8 and 1979-82 (fig 2), parallel to a shift towards relatively more infants born extremely preterm. The gestational age in the very preterm group deceased by two weeks on average between these two periods and then became fairly constant. In fact, no single infant with infantile hydrocephalus was born extremely preterm in the 12 year period $1967-78,{ }^{3}$ as compared with 15 in the period 1979-90. We feel that the first sharp rise could be explained primarily by an increase in the survival of extremely preterm infants, a new group with a particular high risk of IVH resulting in infantile hydrocephalus. In the next period, 1983-6, there was a further slight increase in infantile hydrocephalus prevalence among very preterm infants without any fall in mean gestational age, thereby indicating that the continuous increase in survivors was now taking place at the expense of additional infantile hydrocephalus cases. The slight decrease in the last period 1987-90 could indicate a better outcome in survivors, even if there was a simultaneous slight decrease in survival rate. A similar pattern of unchanging infantile hydrocephalus prevalence in preterm children was seen in a nationwide Swedish survey of shunt requiring low birthweight infants with infantile hydrocephalus born at $<35$ weeks' gestation in the 10 year period $1979-88 .^{8}$ At the same time the increasing prevalence of cerebral palsy in very preterm children also levelled off. ${ }^{15}$ Taken as a whole, this gives rise to the hope of decreasing rates of neuroimpairment among survivors of very preterm birth.

Among infants born at term, there is less future potential for a decrease in infantile hydrocephalus prevalence. Most full term cases of infantile hydrocephalus are of prenatal origin and advanced neonatal intensive care can scarcely result in anything but more survivors. Fetal hydrocephalus is now frequently diagnosed intrauterinely and such infants are delivered by elective caesarean section near to term. In our experience, infants with infantile hydrocephalus born moderately preterm form a transitional category between those born very preterm and full term, in terms of both aetiology and outcome in survivors.

The origin of infantile hydrocephalus is the decisive factor when it comes to outcome in both term and preterm children. This has previously been underlined ${ }^{1011}$ and is confirmed by the results of this study. It is therefore essential to analyse the type and degree of the pathological condition behind infantile hydrocephalus. In this respect, very preterm children with IVH and term children with severe maldevelopments of the CNS were found to be the most multi-impaired groups.

The trend in infantile hydrocephalus prevalence were identical when calculated per 1000 live births and per 1000 neonatal survivors, thereby indicating no shift from stillborn to neonatally dead infants with infantile hydrocephalus. Five per cent, or 12 infants with infantile hydrocephalus, died within the first month of life, all 12 had congenital infantile hydrocephalus with major malformations of such a scope that they led directly or indirectly to neonatal death. The deaths were evenly distributed over the birth year periods. It is very unlikely that more advanced neonatal care could have changed the poor prognosis in these infants. On the other hand, postnatal deaths up to 2 years of age, which occurred in 18 infants, decreased significantly over time and in parallel with an increasing proportion of children with major additional impairments. The enhanced postnatal survival of children with infantile hydrocephalus thus resulted in an increased prevalence of infantile hydrocephalus at higher ages and also in an increased proportion with additional disabilities.

Multi-impairments were seen especially frequently in the very preterm group in which every infant had an average of two major neuroimpairments in addition to infantile hydrocephalus. The most frequently seen was cerebral palsy, present in no fewer than $73 \%$ of very preterm infants with infantile hydrocephalus. The association between cerebral palsy and perinatal IVH was extremely strong. Of a total of 33 very preterm infants with IVH, born in 1983-90, 25 also had cerebral palsy, all of which were spastic forms. As intracranial bleedings resulting in infantile hydrocephalus are known to precede the appearance of periventricular haemorrhage and other brain parenchyma infarctions resulting in cerebral palsy, ${ }^{16} 17$ the data from this study also clearly indicate a perinatal/neonatal origin of cerebral palsy in these children. This is well in line with our experience from patterns in the changing panorama of cerebral palsy in Sweden through four decades. ${ }^{15}$

In the present dynamic and changing world of modern active neonatology, which is continuing to enhance survival, indicators of the long term effects are needed. Our experience supports the theory that valuable, rapidly available feedback to neonatologists can be obtained from a systematic follow up of 
infantile hydrocephalus epidemiology. The main conclusion from this study is that, even though the healthy survivors of very preterm birth far outnumber the impaired ones, the very satisfactory increase in neonatal and postnatal survival has a reverse: the accumulating cohort of impaired children. Society is responsible for the welfare work needed by these children and their parents.

This investigation was supported by grants from the Folke Bernadotte and Sven Jerring Foundations.

1 Hagberg B, Hagberg G, Olow I. Gains and hazards of intensive neonatal care: an analysis from Swedish cerebral

2 Fernell E, Hagberg G, Hagberg B. Infantile hydrocephalus - the impact of enhanced preterm survival. Acta Paediatr Scand 1990; 79: 1080-6.

3 Fernell E, Hagberg B, Hagberg G, von Wendt L. Epidemiology of infantile hyrocephalus in Sweden. I Birth prevalence and general data. Acta Paediatr Scand 1986; 75: 975-81.

4 Sjörgen I. Echo-encephalography in pediatric practice with special regard to measurement of the ventricular size. Acto Paediatr Scand 1967; 66 (suppl): 178.

5 Bax M. Terminology and classification of cerebral palsy. Dev Med Child Neurol 1964; 6: 295-307.

6 Armitage P. Statistical methods in medical research. London: Blackwell Scientific Publications, 1974: 363-5.

7 Davies PA. Infants of very low birth weight. An appraisal of some aspects of their present neonatal care and of their later prognosis. In: Hull $\mathrm{D}$, ed. Recent advances in paediatrics. London: Churchill Livingstone, 1976: 89-128.
8 Fernell E, Hagberg G, Hagberg B. Infantile hydrocephalus in preterm, low-birth-weight infants - a nationwide Swedish cohort study 1979-1988. Acta Paediatr 1993; 82 45-8.

9 Wiklund L-M, Uvebrant P, Flodmark O. Morphology of cerebral lesions in children with congenital hemiplegia: a study with computed tomography. Neuroradiology 1990; 32: 179-86.

10 Fernell E, Hagberg B, Hagberg G, von Wendt L Epidemiology of infantile hydrocephalus in Sweden. II. Opidemiology of infantile hydrocephalus in Sweden. II. 76: $411-7$.

11 Fernell E, Hagberg B, Hagberg G, von Wendt L Epidemiology of infantile hydrocephalus in Sweden. III Origin in preterm infants. Acta Paediatr Scand 1987; 76: 418-23.

12 Papile L-A, Burstein J, Burstein R, Koffler H. Incidence and evolution of subependymal and intraventricular hemorrhage: a study of infants with birth weights less than 1,500 g. F Pediatr 1978; 92: 529-34.

13 Bejar R, Curbelo V, Coen RW, Leopold G, James H, Gluck L. Diagnosis and follow-up of intraventricular and intracerebral hemorrhages by ultrasound studies of intracerebral hemorrhages by ultrasound studies of infant's brain through the

14 Philip AGS, Allan WC, Tito AM, Wheeler LR. Intraventricular hemorrhage in preterm infants: declining incidence in the 1980s. Pediatrics 1989; 84: 797-801.

15 Hagberg B, Hagberg G, Olow I. The changing panorama of cerebral palsy in Sweden. VI. Prevalence and origin during the birth year period 1983-1986. Acta Paediat 1993; 82: 387-93.

16 Cooke RWI. Early and late cranial ultrasonographic appearances and outcome in very low birth weight infants. Arch Dis Child. 1987; 67: 931-7.

17 Volpe JJ. Intraventricular hemorrhage in the premature infant - current concepts. Part I. Ann Neurol 1989; 25: $3-11$ 